\title{
Successes and lessons learned from a community urology regional service
}

Paul R. Martin, MD

Cite as: Can Urol Assoc J 2019 June 27; Epub ahead of print. http://dx.doi.org/10.5489/cuaj.6092

Published online June 27, 2019

$* * *$

Technology in healthcare is rapidly catching up to other industries and the rate of change does not appear to be slowing down. Although most of us still have a Fax number and many have paper charts, system wide adoption Electronic Medical Records, Hospital Information Systems, and other emerging technologies have led to many improvements in patient care. With these advancements come new challenges. I believe there is a need and a patient demand for improved regional integration and coordination of care delivery. Urologists in Canada are well positioned to take advantage of these technological changes to improve care for our patients and to improve our job satisfaction.

The government of Ontario has recently proposed a major overhaul to the structure of healthcare delivery in the province by creating “Ontario Health Teams” which may serve up to 300,000 people each. This change has been touted as the most significant organizational change in 50 years and is consistent with national trends towards healthcare integration. ${ }^{1}$

One stated rationale for the change is to improve coordination of care, mostly primary and home care, but also specialist care. It doesn't appear that the specialist role in an OHT has been defined, and this could be an opportunity for urologists to be proactive in defining our own role. In Urology, while there has been a positive, evidence-based push for regionalization of specialized surgeries, I believe that regional coordination of the "routine" care that we provide (consultations, diagnostic procedures) could also have a significant benefit for the overall urological health of our patients.

There are 716 Urologists in Canada in 2018, or roughly one urologist for every 50K population. Compared to 2241 General Surgeons, or three surgeons for every $50 \mathrm{~K}$. ${ }^{2}$ There are several cities in Canada that don't have a population to support an "in-house" urologist. It is difficult for many patients to travel. Many of our patients are elderly and frail, or don't have the means, or the support to travel out of town for surgery. By using new technology, we now can provide better care for these patients. 
I thought it might be helpful to share some of our successes and challenges in establishing a regional care model in Southwestern Ontario so that others might be able to use this information to develop partnerships and improve regional delivery of care for urology patients.

In 2014 there were three urologists in Sarnia and two urologists in Chatham (combined population of 225,000). Both urologists in Chatham planned to retire from practice. Due to financial constraints at the time, the Hospital was not able to modernize the local urology program. Working with stakeholders in Sarnia and Chatham we developed a program where the urologists in Sarnia would provide elective and emergency care for the patients in Chatham and we would travel to Chatham from time to time for outpatient clinics. ${ }^{3}$

There can be benefits to travelling out of town to see patients. The benefits to patients are clear if there isn't a local urologist: there is a huge cost in time and money for many patients to attend a medical visit out of town. For the surgeon, travelling to other hospitals to see patients may seem like a nuisance. However, there are benefits to the physician as well. For example, these hospitals provide resources and clinic time and can free up your office staff. Your own institution may have limited space for procedures such as BCG, uroflow, and cystoscopy, and another site can offer more resources. We have found that hospitals are eager to have a urologist from out of town come care for their patients. Additional remuneration for on-call coverage might also be available.

We have also found that telemedicine has added significant value. The biggest limiting factor for telemedicine in urology is the inability do perform a digital rectal examination! Most follow-up encounters can be done via telemedicine. There are also several types of patients who can be seen for initial consultation via telemedicine such as: microscopic hematuria, renal colic, renal mass, erectile dysfunction, OAB/SUI. There are additional benefits such as telemedicine billing premiums. The patient is registered at another hospital and is assisted by a nurse. The urologist can be at home or at the office and documents on his/her own EMR. The encounter occurs via an online secure portal through the Ontario Telemedicine Network (OTN). ${ }^{4}$ Another strategy that we have used is eConsults. eConsults can be performed on your own time, between cases for example, and generally take about 10 minutes. Reimbursement is \$200 per hour in Ontario through OTN. eConsults can be requested by any registered practitioner in the province.

While the consultation and diagnostic program had some success, there are problems for which we were unable to find a solution. For example, some General Surgery and Gynecology patients had to go out of town if there was a higher risk of ureteral injury for their surgery. We tried to have a local Nurse Practitioner offer some clinical assessments, but the benefits did not outweigh the costs and it did not decrease the need for patient or physician travel. Most importantly it is very difficult for some patients to travel long distances for surgery. It became clear over time that patients in Chatham will be best served by having a local urologist, particularly for surgical procedures and emergency conditions. ${ }^{5}$ Thankfully the population is 
large enough to support a quality local urology program, and the hospital is actively recruiting. Other smaller cities will continue to rely on regional models of care. I am hopeful that our future will involve increased formal and informal collaboration between regional groups of urologists with models such as shared call, shared EMR, community sub-specialization, telemedicine, teleconference, and other strategies.

Urologists in Canada can leverage new technologies and models of regional care delivery in order to provide the best possible care for our patients. I am sure that many of us have tried these and other strategies to improve care for our patients and I would encourage others to share their experience.

\section{References}

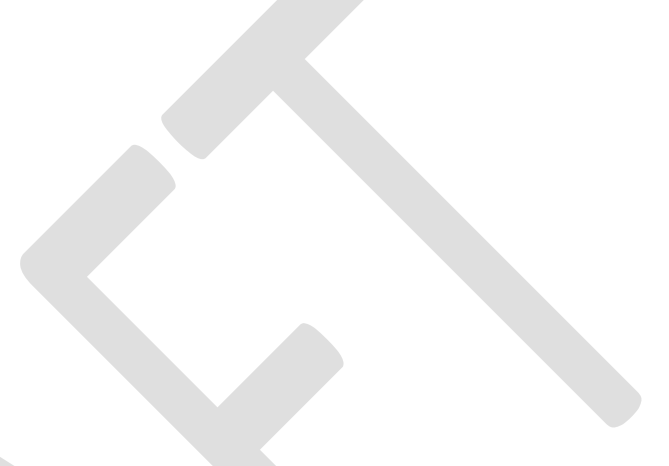

1. Ontario Health Teams: Guidance for Health Care Providers and Organizations. Ministry of Health. http://health.gov.on.ca/en/pro/programs/connectedcare/oht/docs/guidance_doc_en .pdf

2. 2017 CMA Workforce Survey. Canadian Medical

Association. https://www.cma.ca/sites/default/files/2019-01/urology-e.pdf

3. Press Release 07/10/2015. Chatham-Kent Health

Alliance. http://www.ckha.on.ca/chatham-kent-health-alliance-implements-innovativeand-sustainable-urology-solution-chatham-kent

4. Ontario Telemedicine Network Specialist Portal. https://otnhub.ca/physicians/\#specialist

5. Morrison, Tom. Chatham-Kent Health Alliance hopes to reintroduce urology services. Published on: January 25, 2019 https://www.chathamdailynews.ca/news/localnews/chatham-kent-health-alliance-hopes-to-reintroduce-urology-services 